\title{
Analysis and Evaluation of the Main Ecoclimatic Conditions from Romanian Vineyards
}

\author{
Florin Dumitru BORA ${ }^{1 *}$, Ionica DINA², Maria ILIESCU³ ${ }^{3}$ Gabi ZALDEA ${ }^{4}$, Ionela Cătălina GUȚA ${ }^{5}$ \\ ${ }^{1}$ Research Station for Viticulture and Enology, Targu Bujor, Romania, ${ }^{2}$ Research Station for Viticulture \\ and Enology, Murfatlar, Romania, ${ }^{3}$ Research Station for Viticulture and Enology, Blaj, Romania, \\ ${ }^{4}$ Research Station for Viticulture and Enology, Iași, Romania, ${ }^{5}$ National Research \& Development \\ Institute for Biotechnology in Horticulture Ștefănești-Argeș, Romania. \\ *)Corresponding author, e-mail: boraflorindumitru@gmail.com
}

BulletinUASVM Horticulture 73(2) / 2016

Print ISSN 1843-5254, Electronic ISSN 1843-5394

DOI:10.15835/buasvmcn-hort:12122

\begin{abstract}
Vines act as indicators in relation ecoclimatic changes and they are particularly sensitive to temperature changes. Temperature is a limiting factor and define the distribution area for all plants. The main objective of this paper is to present the climatic conditions such as temperature, insolation, rainfall and cloudiness of Romanian main vineyards (Dealu Bujorului, Murfatlar, Târnave, Iași, Ștefănești-Argeși). The thermal balance with the highest values was recorded in Murfatlar vineyard having a value of $5288\left(\sum t g\right)$, the active thermal balance $\left(\sum t a\right) 4815$, and the useful thermal balance $\left(\sum t u\right) 2514$. Insolation and precipitations are in normal parameters for viticulture. The highest values of the real heliothermic index $\left(\mathrm{IH}_{r}\right)$, hydrothermal coefficient $(\mathrm{CH})$ and wine bioclimatic index $\left(\mathrm{I}_{\mathrm{bcv}}\right)$ were recorded in Murfatlar vineyard $\left(\mathrm{IH}_{\mathrm{r}}=4.1 ; \mathrm{CH}=0.8\right.$ and $\left.\mathrm{I}_{\mathrm{bcv}}=11.4\right)$. The result show that the ecoclimatic conditions from the studied vineyards are favorable, that makes possible the production of both red and white wines of high quality in these areas.
\end{abstract}

Keywords: climatic conditions, insolation, rainfall, temperature

\section{Introduction}

Temperature is a limiting factor, it works by limiting the distribution area for all plants, and also for viticulture.

\section{Aims and objectives}

The main objective of this paper is to present the climatic conditions such as temperature, insolation, rainfall and cloudiness of Romanian main vineyards (Dealu Bujorului, Murfatlar, Târnave, Iași, Ștefănești-Argeși).

\section{Materials and methods}

The climatic data conditions were obtained from the Agro Expert system (meteorological station for each vine area studied) and also from the National Meteorological Administration. Based on their specific formulas (Țârdea and Dejeu, 1995; Pop, 2010), ecoclimatic indicators were determined, important for the growth and the fruition of vines, such as global thermal balance $\left(\sum \mathrm{t}^{\circ} \mathrm{g}\right)$; active thermal balance $\left(\sum \mathrm{t}^{\circ} \mathrm{a}\right)$; useful thermal balance $\left(\sum \mathrm{t}^{\circ} \mathrm{u}\right)$; thermal coefficient $\left(C_{t}\right)$; annual and monthly rainfall amount; amount of hours of sunshine ( $\mathrm{ir}$ ) and real sunburn coefficient $\left(C_{i}\right)$. To get a broader image on how climatic factors influence the growth and fruition of vines, the heliothermic index (HI), hydrothermal coefficient $(\mathrm{CH})$ and bioclimatic index $\left(\mathrm{I}_{\mathrm{bcv}}\right)$ were calculated (Bora et al., 2015).

\section{Results and discussion}

The length of the vegetation period is within the normal cultivation limits of vines, 185 days vineyard Dealul Bujorului, 198 days vineyard of Murfatlar, 183 days vineyard Târnava, 174 days vineyard Iași and 184 days vineyard ȘtefăneștiArgeș. In the experimental years 2010-2014, 
the thermal balance with the highest values was recorded in Murfatlar vineyard having a value of $5288\left(\sum t g\right)$, the active thermal balance $\left(\sum t a\right)$ 4815 , and the useful thermal balance $\left(\sum t u\right)$ 2514. Insolation and precipitations are in normal parameters for viticulture. The highest values of the real heliothermic index $\left(\mathrm{IH}_{\mathrm{r}}\right)$, hydrothermal coefficient $(\mathrm{CH})$ and wine bioclimatic index $\left(\mathrm{I}_{\mathrm{bcy}}\right)$ were recorded in Murfatlar vineyard $\left(\mathrm{IH}_{\mathrm{r}}=4.1\right.$; $\mathrm{CH}=0.8$ and $\mathrm{I}_{\mathrm{bcv}}=11.4$ ).

\section{Conclusion}

The result of this research show that the ecoclimatic conditions from the studied vineyards are favorable, that makes possible the production of both red and white wines of high quality in these areas.

Tab. 1. Climate data from studied areas; period of 2010-2015

\begin{tabular}{|c|c|c|c|c|c|c|}
\hline \multirow{2}{*}{ Area } & \multirow{2}{*}{\multicolumn{2}{|c|}{ Studied elements }} & \multirow{3}{*}{$\begin{array}{c}\begin{array}{c}\text { Average } \\
\text { values }\end{array} \\
185\end{array}$} & \multicolumn{2}{|c|}{ Extreme limits } & \multirow{3}{*}{$\begin{array}{c}\text { Optimal values } \\
\text { for viticulture } \\
160-180 \\
\end{array}$} \\
\hline & & & & Min. & Max. & \\
\hline \multirow{12}{*}{$\begin{array}{l}\text { Vineyard } \\
\text { Dealu } \\
\text { Bujorului }^{1}\end{array}$} & The vegetation period & Days & & 183 & 194 & \\
\hline & \multirow{4}{*}{ Thermal balance } & Global $\left(\sum \mathrm{t}^{\circ} \mathrm{g}\right)$ & 3480 & 3354 & 3562 & $2700-4000$ \\
\hline & & Active $\left(\sum \mathrm{t}^{\circ} \mathrm{a}\right)$ & 3385 & 3316 & 3398 & $2500-3800$ \\
\hline & & Useful $\left(\sum \mathrm{t}^{\circ} \mathrm{u}\right)$ & 1681 & 1654 & 1702 & $1000-1800$ \\
\hline & & Thermal coefficient $\left(C_{t}\right)$ & 18.2 & 17.4 & 18.9 & $16-19$ \\
\hline & \multirow{2}{*}{ Insolation (hours) } & Real $\left(\sum\right.$ ir $)$ & 1874 & 1851 & 1896 & $1200-1800$ \\
\hline & & Coefficient of insolation $\left(\mathrm{C}_{i}\right)$ & 8.56 & 8.32 & 8.77 & - \\
\hline & \multirow{2}{*}{ Precipitations (mm) } & Total annual ( $\left.\sum p p\right)$ & 505.0 & 501.0 & 513.0 & $500-700$ \\
\hline & & Coefficient of precipitation $(\mathrm{Cp})$ & 1.20 & 1.10 & 1.50 & - \\
\hline & \multirow{3}{*}{$\begin{array}{l}\text { The interaction of } \\
\text { climatic factors }\end{array}$} & Real heliothermic Index $\left(\mathrm{IH}_{\Psi}\right)$ & 2.38 & 2.16 & 2.41 & $1.35-2.70$ \\
\hline & & Hydrothermal coefficient $(\mathrm{CH})$ & 1.06 & 1.03 & 1.10 & 0.7 \\
\hline & & Bioclimatic index $\left(\mathrm{I}_{\text {a }}\right)$ & 8.01 & 8.00 & 8.06 & 5 \\
\hline \multirow{12}{*}{$\begin{array}{l}\text { Vineyard } \\
\text { Murfatlar }^{2}\end{array}$} & The vegetation period & Days & 198 & 189 & 212 & $160-180$ \\
\hline & \multirow{4}{*}{ Thermal balance } & Global $\left(\sum \mathrm{t}^{\circ} \mathrm{g}\right)$ & 5288 & 4853.8 & 5500.9 & $2700-4000$ \\
\hline & & Active $\left(\sum \mathrm{t}^{\circ} \mathrm{a}\right)$ & 4815.6 & 4509.1 & 5033.1 & $2500-3800$ \\
\hline & & Useful $\left(\sum \mathrm{t}^{\circ} \mathrm{u}\right)$ & 2514.0 & 2299.1 & 2600.2 & $1000-1800$ \\
\hline & & Thermal coefficient $\left(C_{t}\right)$ & 24.32 & 22.34 & 25.67 & $16-19$ \\
\hline & \multirow{2}{*}{ Insolation (hours) } & $\operatorname{Real}\left(\sum\right.$ ir $)$ & 1637.6 & 1973.1 & 1435.1 & $1200-1800$ \\
\hline & & Coefficient of insolation $\left(\mathrm{C}_{\mathrm{i}}\right)$ & 8.27 & 9.3 & 7.32 & - \\
\hline & \multirow{2}{*}{ Precipitations (mm) } & Total annual ( $\left.\sum p p\right)$ & 568.7 & 326.8 & 710.3 & $500-700$ \\
\hline & & Coefficient of precipitation $(\mathrm{Cp})$ & 2.04 & 1.26 & 2.81 & - \\
\hline & \multirow{3}{*}{$\begin{array}{l}\text { The interaction of } \\
\text { climatic factors }\end{array}$} & Real heliothermic Index $\left(\mathrm{IH}_{\uparrow}\right)$ & 4.1 & 3.4 & 4.9 & $1.35-2.70$ \\
\hline & & Hydrothermal coefficient $(\mathrm{CH})$ & 0.80 & 0.5 & 1.2 & 0.7 \\
\hline & & Bioclimatic index $\left(\mathrm{I}_{\text {a }}\right)$ & 11.4 & 6.4 & 17.8 & 5 \\
\hline \multirow{12}{*}{$\begin{array}{l}\text { Vineyard } \\
\text { Tarnava }^{3}\end{array}$} & The vegetation period & Days & 183 & 166 & 192 & $160-180$ \\
\hline & \multirow{4}{*}{ Thermal balance } & Global $\left(\sum \mathrm{t}^{\circ} \mathrm{g}\right)$ & 3449 & 3186 & 3683 & $2700-4000$ \\
\hline & & Active $\left(\sum \mathrm{t}^{\circ} \mathrm{a}\right)$ & 3347 & 3085 & 3546 & $2500-3800$ \\
\hline & & Useful $\left(\sum \mathrm{t}^{\circ} \mathrm{u}\right)$ & 1556 & 1365 & 1875 & $1000-1800$ \\
\hline & & Thermal coefficient $\left(C_{t}\right)$ & 17.9 & 16.9 & 19,2 & $16-19$ \\
\hline & \multirow{2}{*}{ Insolation (hours) } & $\operatorname{Real}\left(\sum\right.$ ir $)$ & 1896.2 & 1558 & 2402 & $1200-1800$ \\
\hline & & Coefficient of insolation $\left(C_{i}\right)$ & 7.55 & 6.12 & 8.31 & - \\
\hline & \multirow{2}{*}{ Precipitations (mm) } & Total annual $\left(\sum p p\right)$ & 580.5 & 349.5 & 698.0 & $500-700$ \\
\hline & & Coefficient of precipitation (Cp) & 2.10 & 1.38 & 2.65 & - \\
\hline & \multirow{3}{*}{$\begin{array}{l}\text { The interaction of } \\
\text { climatic factors }\end{array}$} & Real heliothermic Index $\left(\mathrm{IH}_{\downarrow}\right)$ & 2.2 & 1.7 & 3.2 & $1.35-2.70$ \\
\hline & & Hydrothermal coefficient (CH) & 1.1 & 0.8 & 1.3 & 0.7 \\
\hline & & Bioclimatic index $\left(\mathrm{I}_{\mathrm{b}}\right)$ & 7.2 & 4,3 & 9.9 & 5 \\
\hline
\end{tabular}




\begin{tabular}{|c|c|c|c|c|c|c|}
\hline \multirow{12}{*}{$\begin{array}{l}\text { Vineyard } \\
\text { Iași }^{4}\end{array}$} & $\begin{array}{c}\text { The vegetation period } \\
\text { (days) }\end{array}$ & Days & 174 & 164 & 189 & $160-180$ \\
\hline & \multirow{4}{*}{ Thermal balance } & Global $\left(\sum \mathrm{t}^{\circ} \mathrm{g}\right)$ & 3371 & 3219 & 3653 & $2700-4000$ \\
\hline & & Active $\left(\sum \mathrm{t}^{\circ} \mathrm{a}\right)$ & 3269 & 3077 & 3596 & $2500-3800$ \\
\hline & & Useful $\left(\sum \mathrm{t}^{\circ} \mathrm{u}\right)$ & 1583 & 1427 & 1856 & $1000-1800$ \\
\hline & & Thermal coefficient $\left(C_{t}\right)$ & 18.8 & 16,3 & 20.5 & $16-19$ \\
\hline & \multirow{2}{*}{ Insolation (hours) } & Real ( $\sum$ ir $)$ & 1442 & 1337 & 1513 & $1200-1800$ \\
\hline & & Coefficient of insolation $\left(C_{i}\right)$ & 8.26 & 7.44 & 8.70 & - \\
\hline & \multirow{2}{*}{ Precipitations (mm) } & Total annual ( $\left.\sum p p\right)$ & 557.2 & 365.5 & 674.3 & $500-700$ \\
\hline & & Coefficient of precipitation (Cp) & 2.08 & 1.04 & 3.00 & - \\
\hline & \multirow{3}{*}{$\begin{array}{l}\text { The interaction of } \\
\text { climatic factors }\end{array}$} & Real heliothermic Index $\left(\mathrm{IH}_{\psi}\right)$ & 2.3 & 2.0 & 2.8 & $1.35-2.70$ \\
\hline & & Hydrothermal coefficient $(\mathrm{CH})$ & 1.10 & 0.8 & 1.6 & 0.7 \\
\hline & & Bioclimatic index $\left(\mathrm{I}_{\text {bev }}\right)$ & 8.7 & 5.4 & 16.3 & 5 \\
\hline \multirow{12}{*}{$\begin{array}{l}\text { Vineyard } \\
\text { Ștefanești }\end{array}$} & The vegetation period & Days & 184.33 & 179 & 202 & $160-180$ \\
\hline & \multirow{4}{*}{ Thermal balance } & Global $\left(\sum \mathrm{t}^{\circ} \mathrm{g}\right)$ & 3529.10 & 3279.1 & 3942.1 & $2700-4000$ \\
\hline & & Active $\left(\sum \mathrm{t}^{\circ} \mathrm{a}\right)$ & 3348.36 & 2690.5 & 3907.5 & $2500-3800$ \\
\hline & & Useful $\left(\sum \mathrm{t}^{\circ} \mathrm{u}\right)$ & 1644.22 & 1290.9 & 1921.1 & $1000-1800$ \\
\hline & & Thermal coefficient $\left(C_{t}\right)$ & 18.01 & 14.86 & 19.5 & $16-19$ \\
\hline & \multirow{2}{*}{ Insolation (hours) } & Real $\left(\sum\right.$ ir $)$ & - & - & - & $1200-1800$ \\
\hline & & Coefficient of insolation $\left(C_{i}\right)$ & - & - & - & - \\
\hline & \multirow{2}{*}{ Precipitations (mm) } & Total annual $\left(\sum p p\right)$ & 667.4 & 475.5 & 959.3 & $500-700$ \\
\hline & & Coefficient of precipitation (Cp) & 2.40 & 1.6 & 7.8 & - \\
\hline & \multirow{3}{*}{$\begin{array}{l}\text { The interaction of } \\
\text { climatic factors }\end{array}$} & Real heliothermic Index $\left(\mathrm{IH}_{\uparrow}\right)$ & - & - & - & $1.35-2.70$ \\
\hline & & Hydrothermal coefficient $(\mathrm{CH})$ & 1.24 & 0.80 & 2.1 & 0.7 \\
\hline & & Bioclimatic index $\left(\mathrm{I}_{\text {W }}\right)$ & - & - & - & 5 \\
\hline
\end{tabular}

Acknowledgments. This paper was published under the frame of the Romanian Ministry of Agriculture and Rural Development, project ADER no. 14.2.2. "Quantitative studies on assessment and monitoring contaminants, on the chain of viticulture and winemaking to minimize the amount of pesticides and heavy metals as principal pollutants".

\section{REFERENCES}

1. Bora FD, Pop TI, Bunea CI, Popescu D, Iliescu M, Pop N (2015). The assessment of the main climatic conditions in north-west of Romania for viticulture (1991-2013). Bulletin UASVM Horticulture 72(2):445-447.

2. Pop N (2010). General course of viticulture. Ed. Eikon, Cluj-Napoca.

3. Țârdea C. Dejeu L (1995). Viticulture. Ed. Didactică și Pedagogică, R.A. București. 